\title{
B. ESCOFIER
}

\section{B. LEROUX}

\section{Rotation du sous-espace invariant d'un endomorphisme symétrique de $R^{n}$ par une perturbation symétrique}

Revue française d'automatique, informatique, recherche opérationnelle. Analyse numérique, tome 9, $\mathrm{n}^{0} 1$ (1975), p. 5-8.

$<$ http://www.numdam.org/item?id=M2AN_1975_9_1_5_0>

(C) AFCET, 1975, tous droits réservés.

L'accès aux archives de la revue "Revue française d'automatique, informatique, recherche opérationnelle. Analyse numérique » implique l'accord avec les conditions générales d'utilisation (http://www.numdam.org/legal. php). Toute utilisation commerciale ou impression systématique est constitutive d'une infraction pénale. Toute copie ou impression de ce fichier doit contenir la présente mention de copyright.

\section{Numdam}


R.A.I.R O.

( $9^{\mathrm{e}}$ année, $\mathrm{R}-1,1975, \mathrm{p} 5$ à 8$)$

\title{
ROTATION DU SOUS-ESPACE INVARIANT \\ D'UN ENDOMORPHISME SYMETRIQUE DE $\mathbb{R}^{n}$ PAR UNE PERTURBATION SYMETRIQUE
}

\author{
par B. ESCOFIER* et B. LE ROUX** \\ Communıqué par F ROBERT
}

Résumé - Etant donné deux endomorphismes symetriques $A$ et $C$ de $R^{n}$ tels que $C=A+B$, on se propose de majorer l'angle entre les sous-espacesinvariants de même dimension de $A$ et de $C$ respectivement La borne de cet angle est exprimée en fonction des valeurs propres de $A$ (ou $C$ ) et $B$. Cette borne est optımale pour les sous-espaces invariants assoctés aux plus grandes valeurs propres de $A$ et de $C$

Définition: On appelle ict angle entre 2 sous-espaces de $R^{n}$ l'angle maximum entre un vecteur de l'un des sous-espaces et sa projection orthogonale sur l'autre. [2].

Enoncé des théorèmes :

Solent $A, B, C$ trois endomorphismes symétriques de $R^{n}$ avec $C=A+B$ de valeurs propres respectives, $\alpha_{l}, \beta_{l}, \gamma_{l}$ rangées par ordre décroissant

Théorème 1: Solent $E=\left[v_{1}, \ldots, v_{r}\right]$ et $F=\left[w_{1}, ., w_{r}\right]$ les sous-espaces engendrés respectivement par les $r$ vecteurs propres associés aux $r$ plus grandes valeurs propres de $A$ et de $C$ Solt $\theta$ l'angle entre $E$ et $F$

Sl $\beta_{1}-\beta_{n}<\alpha_{r}-\alpha_{r+1}, \quad$ alors $\theta<\pi / 4$ et $\sin 2 \theta \leqslant \frac{\beta_{1}-\beta_{n}}{\alpha_{r}-\alpha_{r+1}}$.

Sı la condition n'est pas réalısée, l'angle $\theta$ peut atteındre $\pi / 2$.

* IN S A - Département de Mathématıques - Rennes

** UE R de Mathématıques - Unıversité Parıs V

Revue Françaıse d'Automatıque, Informatıque et Recherche Opératıonnelle nº avr1l 1975, R-1 
Théorème 2: Soient $E=\left[v_{k}, \ldots, v_{k+r}\right]$ et $F=\left[w_{k}, \ldots, w_{K+r}\right]$ les sous-espaces engendrés respectivement par les vecteurs propres de $A$ et $C$ associés aux valeurs propres de rang $k, \ldots, k+r$. Soit $\theta$ l'angle entre $E$ et $F$.

$$
\begin{gathered}
\text { Soit } \delta=\inf \quad\left(\alpha_{k-1}, \alpha_{k}\right),\left(\alpha_{k+r}, \alpha_{k+r+1}\right) \\
\text { alors si } J>\beta_{1}-\beta_{n} \text { on } a \quad \sin \theta \leqslant \frac{\beta_{1}-\beta_{n}}{2 \delta-\left(\beta_{1}-\beta_{n}\right)}
\end{gathered}
$$

Si la condition n'est pas réalisée, l'angle $\theta$ peut atteindre $\frac{\pi}{2}$.

Remarques: Si on pose $A=C+(-B)$, les formules en fonction des valeurs propres de $C$ se déduisent de celles-ci en remplaçant les $\alpha_{i}$ par les $\gamma_{i}$.

Démonstration du théorème 1 :

Si $A, B, C$ sont des endomorphismes de $R^{2}$, on démontre que si $\beta_{1}-\beta_{2}<\alpha_{1}-\alpha_{2}$, l'angle entre $\left[V_{1}\right]$ sous espace invariant de $A$ associé à $\alpha_{1}$ et $\left[W_{1}\right]$ sous-espace invariant de $C$ associé à $\gamma$ est inférieur à $\frac{\pi}{4}$ et que.e $\sin 2 \theta \leqslant \frac{\beta_{1}-\beta_{2}}{\alpha_{1}-\alpha_{2}}$

Dans le cas général, on se ramène à l'étude d'endomorphismes de $R^{2}$ en se plaçant dans le plan $[V, W]$ où $V$ et $W$ sont deux vecteurs appartenant respectivement à $E$ et $F$ et faisant entre eux l'angle $\theta$. Cette méthode s'applique seulement si $E$ et $F$ sont des sous-espaces invariants associés aux plus grandes valeurs propres.

\section{Démonstration du théorème 2 :}

Si $P$ (resp. Q) est la projection orthogonale sur $E$ (resp. F), et $\theta$ l'angle entre $E$ et $F$, on a $: \sin \theta=\|(1-Q) P\|$

On utilise un théorème dû à M.M. DAVIS et KAHAN [1] :

Soient $A, B, C$ trois endomorphismes de $R^{n}$ symétriques avec $C=A+B$. Soit $P$ (resp. Q) la projection orthogonale sur un sous-espace invariant de $A$. (resp. C). S'il existe un intervalle $\left[t^{\prime}, t\right] \quad\left(t^{\prime}<t\right)$ et un nombre $\in>0$ tels que le spectre de la restriction de $P A$ à $P R^{n}$ soit contenu dans $\left[t^{\prime}, t\right]$ et celui de la restriction de $(1-\mathrm{Q}) C$ à $(1-\mathrm{Q}) R^{n}$ normale à $Q R^{n}$ soit hors de $\left[t^{\prime}-\epsilon, t+\in\right]$ alors :

$$
\|(1-\mathrm{Q}) P\| \leqslant \frac{\|B\|}{\epsilon}
$$


Avant d'appliquer ce théorème, translations $A$ et $B$ afin de diminuer la norme de $B$. On pose pour :

$$
\begin{gathered}
B^{\prime}=B-\frac{\beta_{1}+\beta_{n}}{2} I \text { d'où }\|B\|=\frac{\beta_{1}-\beta_{n}}{2} \\
A^{\prime}=A+\frac{\beta_{1}+\beta_{n}}{2} I=C-B^{\prime}
\end{gathered}
$$

Notons que $A^{\prime}$ a les mêmes vecteurs propres que $A$. Il faut que les spectres des restrictions de $(1-\mathrm{Q}) C$ à $(1-\mathrm{Q}) R^{n}$ et de $P A^{\prime}$ à $P R^{n}$ soient séparés d'un intervalle de longueur supérieure à un nombre $\epsilon$.

L'intervalle entre les spectres est :

$$
\inf \left\{\left(\gamma_{k-1}-\alpha_{k}-\frac{\beta_{1}+\beta_{n}}{2} \quad,\left(\alpha_{k+r}+\frac{\beta_{1}+\beta_{n}}{2}-\gamma_{k+r+1}\right)\right\}\right.
$$

D'après le théorème de WIELANDT

$$
\gamma_{k-1} \geqslant \alpha_{k-1}+\beta_{n} \text { et } \gamma_{n+r+1} \leqslant \alpha_{k+r+1}+\beta_{1}
$$

Nous pouvons donc choisir pour $\in$ la valeur suivante :

$$
\begin{gathered}
\in=\inf \left\{\left(\alpha_{k-1}-\alpha_{k}-\frac{\beta_{1}+\beta_{n}}{2}\right),\left(\alpha_{k+r}-\alpha_{k+r+1}-\right.\right. \\
\left.\left.-\frac{\beta_{1}-\beta_{n}}{2}\right)\right\}>0 .
\end{gathered}
$$

Remarque : Cette méthode appliquée à l'étude de l'angle entre les sous-espaces invariants de $A$ et de $C$ engendrés par les $r$ premiers vecteurs donne des majorations supérieures à celle du théorème 1 , qui étaient optimales.

\section{CONCLUSION :}

Les bornes pour les perturbations angulaires sont calculées ici pour des sousespaces invariants de dimensions quelconque, ce qui permet de traiter le cas de valeurs propres multiples ou voisines. Les bornes exprimées habituellement en fonction d'une norme de l'endomorphisme perturbation, le sont ici en fonction de ses valeurs propres. Cette forme a une application en analyse des données. Des expériences numériques ont été faites dans ce domaine [3]. Le théorème concernant les perturbations des "sous-espaces invariants intermédiaires" n'est qu'une application directe du théorème de Davis et Kahan. Le théorème concernant les $\mathrm{n}^{\circ}$ avril $1975, \mathrm{R}-1$. 
perturbations des sous-espaces invariants associés aux plus grandes valeurs propres présente plus d'intérêt car les bornes exprimées en fonction des valeurs propres de l'endomorphisme perturbation sont optımales. On en fait ici une démonstration directe, mais ce résultat peut aussi se dédurre d'un deuxième théorème de M.M. Davis et Kahan [1]

\section{BIBLIOGRAPHIE}

[1] CHANDLER-DAVIS et W.M. KAHAN The rotation of elgenvectors by a perturbation (in SIAM J. Numer Anal. Vol. no 1 March 70).

[2] DEMPSTER Elements of continuous multivariate analysis, (Addision Wesiey 1969).

[3] B. ESCOFIER et B. LE ROuX Etude de 3 problèmes de stabllité en analyse factorlelle, Publications de l'Institut de Statıstıque Parıs (à paraître 1974). 